Title : will be set by the publisher

Editors : will be set by the publisher

EAS Publications Series, Vol. ?, 2013

\title{
HD181068: A TRIPLY ECLIPSING SYSTEM WITH INTRINSICALLY VARIABLE RED GIANT COMPONENT
}

\author{
A. Derekas ${ }^{1}$, T. Borkovits ${ }^{21,3}$, J. Fuller ${ }^{4}$, D. Huber ${ }^{5}$ and H. Lehmann ${ }^{6}$
}

\begin{abstract}
We present the analysis of HD 181068 which is one of the first triply eclipsing triple system discovered. Using Kepler photometry, ground based spectroscopic and interferometric measurements, we determined the stellar and orbital parameters of the system. We show that the oscillations observed in the red giant component of the system are tidally forced oscillations, while one of the most surprising results is that it does not show solar-like oscillations.
\end{abstract}

\section{Introduction}

The ultraprecise and continous photometry of Kepler space telescope led to great discoveries not just in the field of exoplanets but in stellar astrophysics too. Among others, it opened a new era in the investigation of multiple star systems, such as discoveries of the first triply eclipsing triple systems, KOI-126 (Carter et al. (2011)) and HD 181068 (Derekas et al. (2011)). The special geometry of these triply (or mutually) eclipsing triple systems, enables us fast and easy determination of further characteristics that otherwise could only be studied with great effort on a long time-scale.

Here we present the analysis of the triply eclipsing hierarchical triple system HD 181068 based on Kepler photometry, ground based high-resolution spectroscopy and interferometry. HD 181068 has a magnitude of $\mathrm{V}=7.1$ and a distance of about $250 \mathrm{pc}$ (van Leeuwen (2007)). It has been previously identified as

\footnotetext{
1 Konkoly Observatory, Research Centre for Astronomy and Earth Sciences, Hungarian Academy of Sciences, H-1121 Budapest, Konkoly Thege M. út 15-17, Hungary; e-mail: derekas@csfk.mta.hu

2 Baja Astronomical Observatory, H-6500 Baja, Szegedi út, Kt. 766, Hungary

${ }^{3}$ ELTE Gothard-Lendület Research Group, H-9700 Szombathely, Szent Imre herceg ut 112, Hungary

${ }^{4}$ Center for Space Research, Department of Astronomy, Cornell University, Ithaca, NY 14853, USA

5 NASA Ames Research Center, MS 244-30,Moffett Field, CA 94035, USA

6 Thüringer Landessternwarte Tautenburg, 07778 Tautenburg, Germany
} 

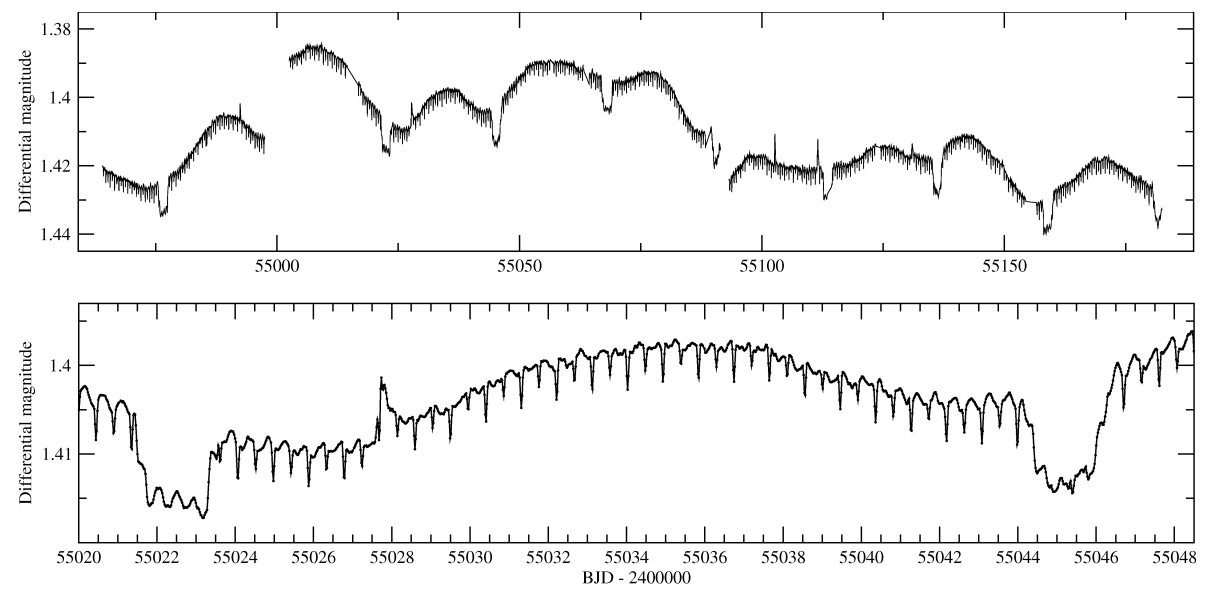

Fig. 1. Kepler-band light curve of HD 181068 from observations in long-cadence mode. Top panel: 218 days of observations, bottom panel: a 28-d segment showing two consecutive deep minima. Figure is taken from Derekas et al. (2011).

a single-lined spectroscopic binary (Guillout et al. (2009)) but there have been no reports of eclipses.

\section{Kepler data}

HD 181068 was observed by the Kepler space telescope in long cadence mode (one point every 29.4 minutes) for the entire mission, and in short cadence mode (time resolution of $58.9 \mathrm{sec}$ ) from Q7-Q16 using a Custom Made Aperture Mask. The light curve is shown in Fig. 1.

The observations reveal a very distinctive light curve. It shows eclipses every $\sim 22.7$ days and slow variations in the upper envelope (top panel in Fig. 1) that are likely caused by ellipsoidal distortion of the primary component $(A)$. There are also very regular and much narrower eclipses (bottom panel in Fig. 1). These minima have alternating depths, corresponding to a close pair ( $B a$ and $B b$ ), with an orbital period of $0.90567 \mathrm{~d}$. The 22.7 -d eclipses all have similar depths, but there are subtle differences between consecutive minima. The true orbital period of the $B a B b$ pair around the $A$ component is $45.5178 \mathrm{~d}$. In addition to the eclipses, there are brightness fluctuations during the long period minima which imply that component $\mathrm{A}$ is also an intrinsic variable star with a mean cycle length close to half the shorter orbital period, indicating tidally-induced oscillations. There were also several flare-like events in the light curve that usually lasted about 6-8 hours. More detailed description of the light curve can be found in Derekas et al. (2011) and Borkovits et al. (2013). 


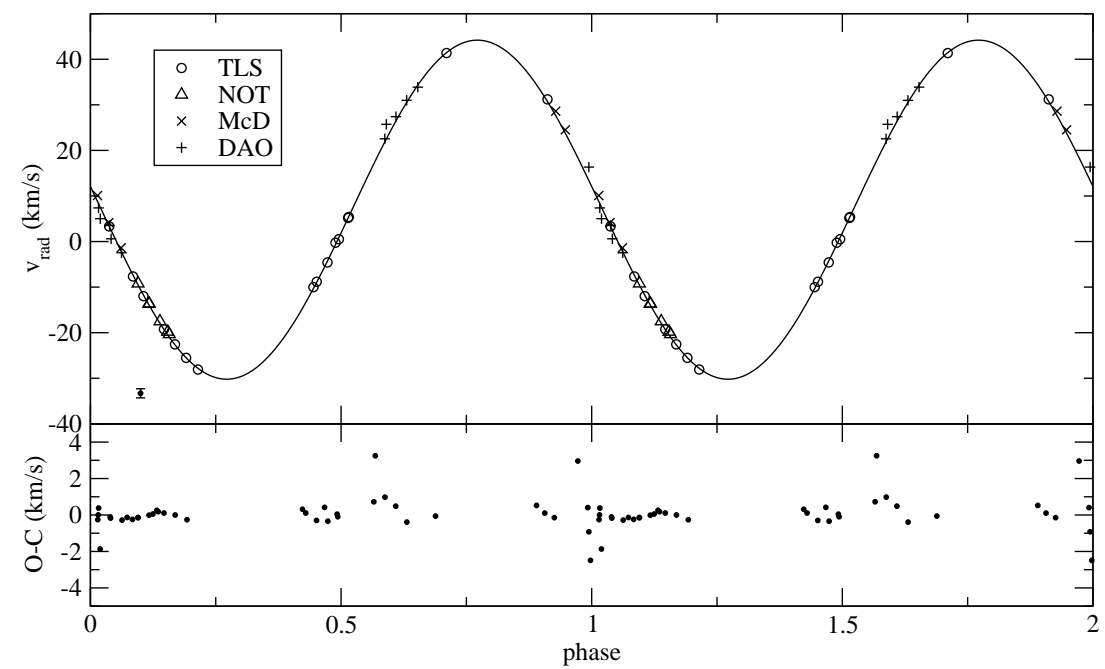

Fig. 2. Phase diagram of the measured RVs. The vertical bar in the lower left corner shows the size of the representative $\pm 1 \mathrm{~km} / \mathrm{s}$ uncertainty. Figure is taken from Derekas et al. (2011).

\section{Spectroscopy}

We obtained 41 high-resolution optical spectra using FIES spectrograph at the Nordic Optical Telescope (NOT), Dominion Astrophysical Observatory (DAO), Thüringer Landessternwarte (TLS) Tautenburg and the $2.7 \mathrm{~m}$ telescope with the Robert G. Tull coudè spectrograph at the McDonald Observatory (McD). As $99.3 \%$ of the light comes from star $A$, we could not find any signiture of the close pair in the spectra. Therefore, the measured radial velocities represent the orbital reflex motion of star $A$ (Fig. 2). We also determined the stellar parameters of the star $A$ by fitting theoretical template spectra from the library of Munari, et al. (2005) to a NOT spectrum. The resulted parameters are listed in Table 1.

\section{Interferometry}

Interferometric observations were performed in three nights in 2010 July, using two different baselines (156.3 $\mathrm{m}$ and $248.1 \mathrm{~m}$ ) of the CHARA Array. Detailed description of the observations can be found in the Support Online Material of Derekas et al. (2011). The corresponding linear limb-darkening coefficient was determined by interpolating the spectroscopically determined values of $\log \mathrm{g}$ and $\mathrm{T}_{\text {eff }}$ in the grid of Claret (2000), yielding $\mu=0.63 \pm 0.02$. The resulting angular diameter of component $A$ is $\theta_{\mathrm{LD}}=0.461 \pm 0.011$ milli-arcsecond. Combining 


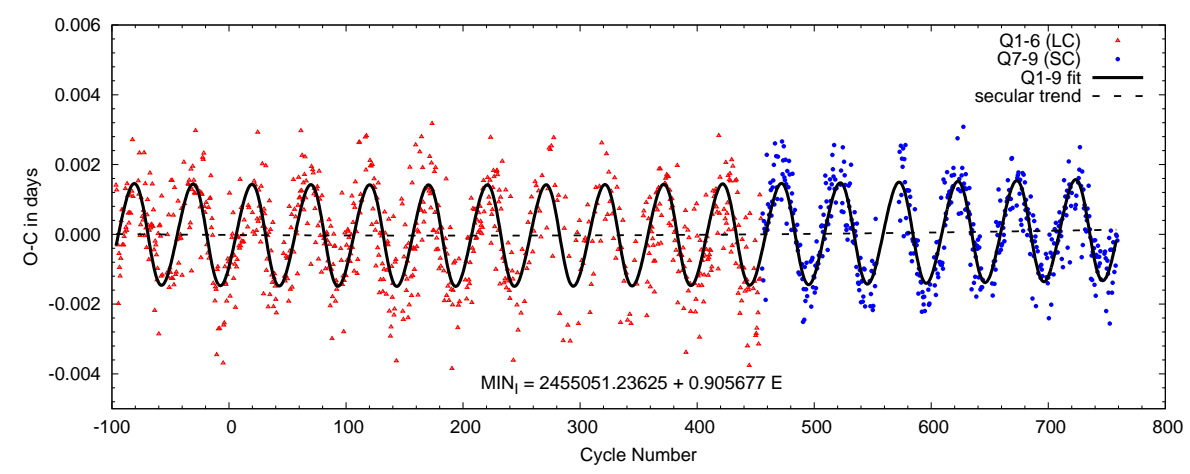

Fig. 3. Eclipse timing variations in the shallow minima. Triangles and circles mark the LC and SC data, respectively. The solid curve stands for the Q1 Q9 fit, while the dashed line represents the weak secular trend (parabolic fit). Figure is taken from Borkovits et al. (2013).

this with the Hipparcos parallax: $4.0 \pm 0.4$ mas, we can calculate the radius as $R_{A}=12.4 \pm 1.3 R_{\odot}$.

\section{Eclipse timing variations and light curve fit}

In order to study the eclipse timing variations (ETV), the following linear ephemeris was calculated for the shallow minima:

$$
M I N_{\text {I-shallow }}[B J D]=2455051.23625+0.905677 \times E,
$$

where $\mathrm{E}$ is the cycle number (Borkovits et al. (2013)). The corresponding ETV diagram is plotted in Fig. 3.

We see a sinusoidal variation with a period identical to the eclipsing period of the wide system. There is also a smaller, long-term variation, that might either be part of a longer period variation, or represent a secular trend, as is the case with several close binary systems.

Combining the measured ETVs with the single-lined radial velocity measurements we could determine masses in a manner equivalent to double-lined spectroscopic binaries. The final parameters are listed in Table 1.

We have also developed a new light curve synthesis code (Borkovits et al. (2013)) that is used to model the triple, mutual eclipses and the effects of the changing tidal field on the stellar surface and the relativistic Doppler-beaming. By combining the stellar masses from the ETV study with the simultaneous light curve analysis we determine the absolute radii of the three stars. Our results indicate that the close and the wide subsystems revolve in almost exactly coplanar and prograde orbits (Fig. 4). We note that the radius of star $A$ from the dynamical model is in excellent agreement with the radius derived from interferometry. 

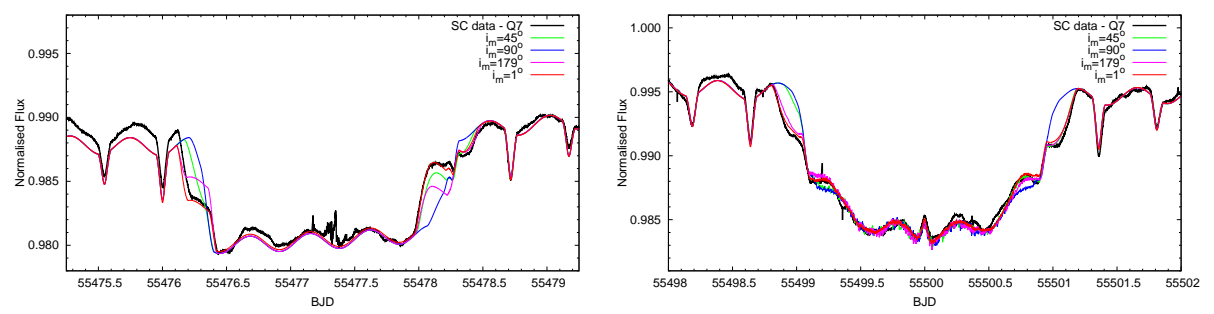

Fig. 4. Synthetic light curves for the outer eclipses. Secondary (left) and primary (right) minima are calculated for different mutual inclinations. Figure is taken from Borkovits et al. (2013).

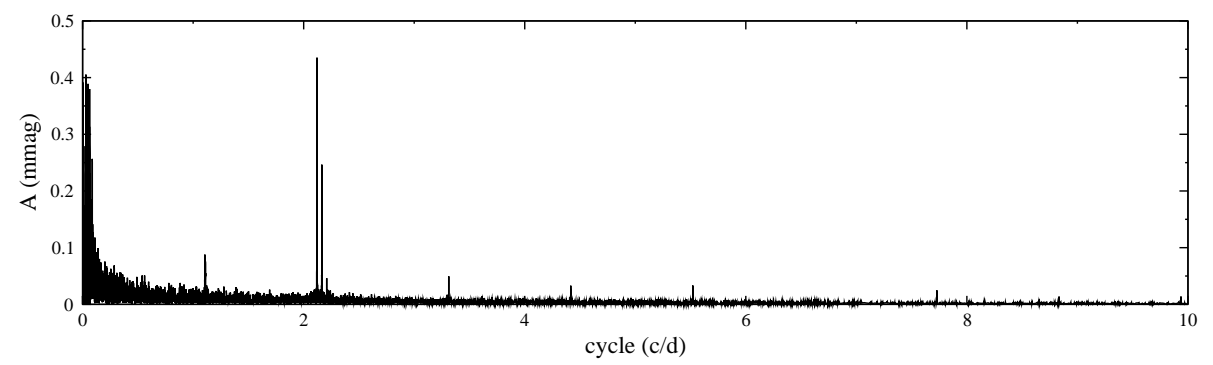

Fig. 5. Fourier spectrum of 11 quarters of long cadence data. Figure is taken from Fuller et al. (2013).

\section{Oscillations}

The light curve (see Fig. 1) shows oscillations with the timescale of the orbital period of the close pair which are visible both outside and during the eclipses and are less obvious to interpret. Fig. 5 shows the Fourier spectrum of the residual light curve after subtracting both the long- and short-period eclipses and rotational variations by using the light curve fit of Borkovits et al. (2013), which resulted in a nearly continuous data set containing the pulsations. For the period analysis, we used Period04 by Lenz \& Breger (2005). In the Fourier-spectra, a number of frequencies were found $\leq 0.1 \mathrm{~d}-1$ indicating long term variability, remnants of the light curve fit, or instrumental effects that are negligible in the present analysis. Significant peaks are listed in Table 2.

The most intriguing result of the period analysis is that $f_{1}$ and $f_{2}$ are linear combinations of the two orbital frequencies, which can be naturally explained by three-body tidal effects (Fuller et al. (2013)). The observed oscillation amplitudes and phases in HD 181068 are in good agreement with theoretical predictions. The angular frequencies are separated by $2 \Omega_{1}$, creating a beat pattern which is in phase near the primary eclipses and occultations (Fuller et al. (2013)). The peaks 
Table 1. Stellar and orbital parameters derived from the combined ETV and synthetic light curve analysis. The numbers in parantheses are the estimated errors in the last digits. Table is taken from Borkovits et al. (2013).

\begin{tabular}{|c|c|c|c|}
\hline \multicolumn{4}{|c|}{ orbital parameters } \\
\hline & \multicolumn{3}{|c|}{ subsystem } \\
\hline & \multicolumn{2}{|c|}{$\mathrm{Ba}-\mathrm{Bb}$} & $\mathrm{A}-\mathrm{B}$ \\
\hline $\bar{P}[\mathrm{~d}]$ & \multicolumn{2}{|c|}{$0.9056768(2)$} & $45.4711(2)$ \\
\hline$T_{\mathrm{MINI}}[\mathrm{BJD}]$ & \multicolumn{2}{|c|}{$2455051.23623(5)$} & $2455499.9962(4)$ \\
\hline$a\left[\mathrm{R}_{\odot}\right]$ & \multicolumn{2}{|c|}{$4.777(39)$} & $90.31(72)$ \\
\hline$e$ & \multicolumn{2}{|c|}{0.0} & 0.0 \\
\hline$\omega$ & \multicolumn{2}{|c|}{-} & - \\
\hline$i[\mathrm{deg}]$ & \multicolumn{2}{|c|}{$86.7(14)$} & $87.5(2)$ \\
\hline$\Delta \Omega[\operatorname{deg}]$ & \multirow{2}{*}{\multicolumn{3}{|c|}{$\begin{array}{c}0.0(5) \\
0.8(14)\end{array}$}} \\
\hline$i_{\mathrm{m}}[\mathrm{deg}]$ & \multicolumn{2}{|c|}{$0.8(14)$} & \\
\hline$q$ & \multirow{2}{*}{\multicolumn{2}{|c|}{$\begin{array}{c}0.95(3) \\
0.3468\end{array}$}} & $0.595(5)$ \\
\hline$L_{\mathrm{sec}} / L_{\mathrm{TOT}}$ & & & 0.0078 \\
\hline \multicolumn{4}{|c|}{ stellar parameters } \\
\hline & $\mathrm{Ba}$ & $\mathrm{Bb}$ & $\mathrm{A}$ \\
\hline \multicolumn{4}{|c|}{ fitted and/or derived parameters } \\
\hline \multicolumn{4}{|c|}{ relative quantities } \\
\hline$r_{\text {pole }}$ & 0.1798 & 0.1664 & 0.1376 \\
\hline$r_{\text {side }}$ & 0.1808 & 0.1672 & 0.1379 \\
\hline$r_{\text {point }}$ & 0.1826 & 0.1687 & 0.1382 \\
\hline$\underline{r}_{b a c k}$ & 0.1822 & 0.1684 & 0.1382 \\
\hline \multicolumn{4}{|c|}{ absolute quantities } \\
\hline$m\left[\mathrm{M}_{\odot}\right]$ & $0.915(34)$ & $0.870(43)$ & $3.0(1)$ \\
\hline$R\left[\mathrm{R}_{\odot}\right]$ & $0.865(10)$ & $0.800(20)$ & $12.46(15)$ \\
\hline$T_{\text {eff }}[\mathrm{K}]$ & $5100(100)$ & $4675(100)$ & $5100(100)$ \\
\hline$L_{\mathrm{bol}}\left[\mathrm{L}_{\odot}\right]$ & $0.447(37)$ & $0.270(27)$ & $92.812(7615)$ \\
\hline $\log g[\mathrm{dex}]$ & 4.53 & 4.58 & 2.73 \\
\hline \multicolumn{4}{|c|}{ fixed quantities } \\
\hline$\overline{k_{2}}$ & 0.020 & 0.020 & 0.033 \\
\hline$\beta$ & 0.32 & 0.32 & 0.32 \\
\hline$A$ & 0.5 & 0.5 & 0.5 \\
\hline$x_{b o l}$ & 0.71476 & 0.71476 & 0.71159 \\
\hline$y_{b o l}$ & 0.13026 & 0.13026 & 0.12561 \\
\hline$x_{K}$ & 0.70835 & 0.70835 & 0.70074 \\
\hline$y_{K}$ & 0.16354 & 0.16354 & 0.16609 \\
\hline
\end{tabular}

frequencies $f_{3}$ and $f_{4}$ correspond to one and two times the orbital frequency of $B a$ and $B b$, and may be caused by the imperfect subtraction of the eclipses or spots on the components. However, they could have a tidal component as well. 
Table 2. The significant peaks from the period analysis of HD 181068. $f_{1}$ and $f_{2}$ are the two pulsation modes, while a number of peaks located $<0.1 \mathrm{~d}^{-1}$ were excluded from the analysis. Table is taken from Fuller et al. (2013).

\begin{tabular}{cccccc}
\hline No. & $\begin{array}{c}\text { Frequency } \\
\left(\mathrm{d}^{-1}\right)\end{array}$ & $\begin{array}{c}\text { Orbital Relation } \\
(\mathrm{mmag})\end{array}$ & Amplitude & Phase & $\mathrm{S} / \mathrm{N}$ \\
\hline$f_{1}$ & 2.1203 & $2\left(\Omega_{23}-2 \Omega_{1}\right) /(2 \pi)$ & 0.44 & 0.0403 & 58 \\
$f_{2}$ & 2.1643 & $2\left(\Omega_{23}-\Omega_{1}\right) /(2 \pi)$ & 0.25 & 0.2889 & 35 \\
$f_{3}$ & 1.1065 & $\Omega_{23} /(2 \pi)$ & 0.08 & 0.2843 & 3.4 \\
$f_{4}$ & 2.2084 & $2 \Omega_{23} /(2 \pi)$ & 0.048 & 0.8719 & 6.8 \\
\hline
\end{tabular}

\subsection{Lack of solar-like oscillations}

Red giant stars are well known to show solar-like oscillations (De Ridder et al. (2009), Chaplin et al. (2011)) which are excited by near-surface convection and nearly all red giants observed by Kepler show detectable oscillations (Huber et al. (2010), Kallinger et al. (2010), Hekker et al. (2011)). The upper panel of Fig. 6 shows the power spectrum of the residual Q1-Q11 long-cadence light curve. For comparison the middle panel shows the power spectrum of KIC4662939, a Helium-core burning red giant with similar fundamental properties as red giant component in HD181068. Despite an increase of the data set length by a factor of three compared to Derekas et al. (2011), the lack of solar-like oscillations in HD181068 $A$ is clearly confirmed. On the other hand, we observe that both stars exhibit a similar decrease of power from low to high frequencies, which is the typical signature of granulation (see Mathur et al. (2011)). This confirms that both stars indeed have similar fundamental properties, but that solar-like oscillations are suppressed in HD181068 A.

\section{Acknowledgements}

This project has been supported by the Hungarian OTKA Grant K83790, KTIA URKUT-10-1-2011-0019 grant, the Lendület-2009 Young Researchers Programme of the Hungarian Academy of Sciences and the European Communitys Seventh Framework Programme (FP7/2007-2013) under grant agreement no. 269194 (IRSES/ASK). AD has been supof Szombathely for support under Agreement No. S-11-1027.

\section{References}

Borkovits T., Derekas, A., Kiss, L. L., et al., 2013, MNRAS, 428, 1656

Carter et al., 2011, Science, 331, 562

Chaplin, W., et al., 2011, Science, 332, 213

Claret, A., 2000, A\&A, 363, 1081

Derekas, A., Kiss, L. L., Borkovits, T., et al., 2011, Science, 332, 216

De Ridder, J., et al., 2009, Nature, 459, 398

Fuller, J., Derekas, A., Borkovits, T., Huber, D., Bedding, T. R., Kiss, L. L., 2013, MNRAS, 429, 2425

Guillout P., et al., A\&A, 504, 829 


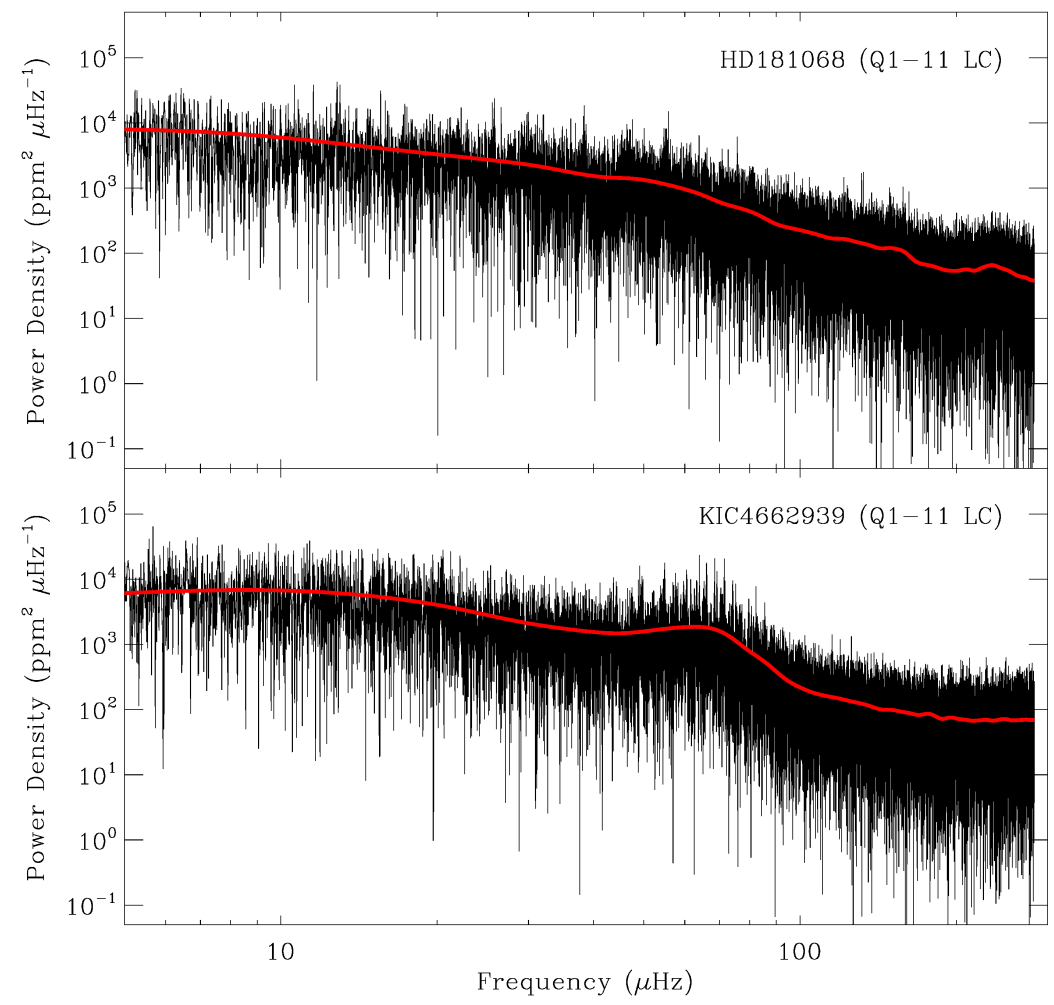

Fig. 6. Top panel: Power spectrum of the Q1-Q11 long-cadence data of HD 181068 after removing eclipses and low frequency variability. The red line shows the power spectrum smoothed with a Gaussian filter with a full-width at half maximum of $5 \mu \mathrm{Hz}$. Bottom panel: panel: Same as top panel but for KIC 4662939. Figure is taken from Fuller et al. (2013)

Hekker, S., et al., 2011, MNRAS, 414, 2594

Huber, D., et al., 2010, ApJ, 723, 1607

Kallinger, T., et al., 2010, A\&A, 522, A1

F. van Leeuwen, 2007. Ap\&SS Library, Vol. 350, Hipparcos, the New Reduction of the Raw Data, Springer, Berlin

Lenz, P., Breger, M., 2005, Comm. Asteroseis., 146, 53

Mathur, S., et al., 2011, ApJ, 741, 119

Munari, S., et al., 2005, Astron. Astrophys. 442, 1127 